\title{
Polarization profiles of polyvinylidene fluoride films polarized by focused electron beam
}

\author{
$D$. Schilling and $K$. Dransield \\ Fakuität für Physik, Universität Konstanz, Postfach 5360,7750 Konstanz, Federal Republic of Germany \\ E. Bihler, K. Holdik, and W. Eisenmenger \\ 1. Physikalisches Institut der Universität Stuttgart, Pfaffenwaldring, 57, 7000 Stuttgart 80, \\ Federal Republic of Germany
}

(Received 18 October 1987; accepted for publication 16 August 1988)

\begin{abstract}
The depth profles of the polarization in films of polyvinylidene fluoride (PVDF) as well as in vinylidene-fiuoride-trifuoroethylene (VDF-TrFE) copolymer films polarized by a focused electron beam were investigated using the piezoelectricaly generated pressure step method. The dominant polarization exhibits a broad maximum inside the film. The position of this maximum depends not only on the energy of the incident electrons but also on the material parameters of the sample. Close to the surface exposed to the electron beam we have in addition observed a small secondary maximum of opposite polarization (amcunting to about 1 $\mathrm{mC} / \mathrm{m}^{2}$ ). A qualitative model is presented for the poling of films of PVDF and its copolymers with TrFE by focused electron beam accounting for most of the observed features. The application of electron beams for the poling of ferroelectric films allows the production of piezoelectric bimorphs. By using a well-focused electron beam also ferroelectric demains of very small lateral dimensions can be created which could become important for ferroelectric data storage.
\end{abstract}

\section{INTRODUCTION}

In view of the many applications of polyvinylidene fuoride (PVDF), the research interest in this material has grown rapidly during the last few years. ${ }^{1-4}$ A number of poling methods for the production of piezo-, pyro-, and ferroelectric PVDF films have been reported: The classical method of electret formation ${ }^{5}$ by the application of a high electric field at elevated temperatures to a PVDF sample, metallized on botn sides, ${ }^{6}$ and the corona poling ${ }^{7}$ are well established.

A new method of poling PVDF reported by Sessler et al. ${ }^{8}$ uses an unfocused monoenergetic electron beam which irradiates a large area of the sample. The special feature of electron-beam poling as explained by Sessler et $a l^{8}$ lies in the finite depth of penetration for electrons into the sample, building up a zone of negative space charges at the characteristic penetration depth (see aiso Ref. 9). This space charge leads to a nonuniform polarization across the sample thickness as first observed by Sessler et al. ${ }^{8}$ and Gross et al ${ }^{10}$ using their weil-known method of laser-induced pressure pulses (LIPP). ${ }^{1 \mathrm{i}-13}$

In this paper we report experimental resuits for poling of PVDF films by a focused electron beam as proposed by Byatt in 1977 (Ref. 14) as a new method for data storage in PVDF based on the ferroelectric properties of this material. We used a focused electron beam to scan across the PVDF film to be poled, and we succeeded also in the electron-beam poling of copolymer films of vinylicene-fivoride-triftuoroethylene (VDF-TrFE).

There are three important differences between poling PVDF by a focused and by a defocused electron beam.

(1) By using a focused electron beam, poling pattems of very small lateral extersion can be created. They can be

\footnotetext{
9) Present address: Standard Electric Lorenz AG, Jorenzstr. 10, 7000 Stuttgart 40, Federal Republic of Germany.
}

made visible by a scanning electron microscope using the method of "potential contrast imaging" as reported elsewhere. ${ }^{15}$ As mentioned above, this possibility to write and read out may be important for memory applications of PVDF, whereas the method of "defocused poling" is quite useful for the production of monomorphs ${ }^{16}$ of large area.

(2) There is another interesting qualitative difference between both poling methods: Whereas in the defocused case the sample surface is charged up uniformly such that all subsequent electrons are slowed down in the planar stopping potential, this does not occur in the same way in the case of poling by a focused electron beam. Here the initial charges are deposited at a small spot deep inside the sample, and therefore the portion of the beam reaching the sample later on is only partly stopped, but mainly deflected laterally, and is still able to penetrate into the sample.

(3) As a consequence of the concentrated energy deposition by the focused electron beam, rapid local heating of the PVDF sample occurs and the temperature can reachaccording to our estimates-values of more than $60^{\circ} \mathrm{C}$, for slow scanning rates (below $0.1 \mathrm{~m} / \mathrm{s}$ ). This local temperature rise may have an accelerating influence on the dipolar switching time in the poling process and may therefore be quite useful for fast recording. If, however, the charge deposition exceeds values of about one electron per $\AA_{A^{2}}^{217}$ chemical changes of the PVDF surface can also occur.

For a detailed investigation of the polarization created by the electron-beam-poling process, we applied the method of piezoelectrically generated pressure step (PPS) developed by Haardt and Eisenmenger ${ }^{18,19}$ to our electron-beampolarized PVDF samples.

This pressure-step-:esponse method is very similar and complementary to the LIPP method mentioned above. ${ }^{20}$ The acoustic pulse response of the LIPP method can be obtained from the PPS response by simply differentiating. For 
a comparison of the various pressure wave methods, see Ref. 13. We used the PPS method to obtain an easy calibration of the signal amplitude with respect to the absolute value of the apparent piezoelectric constant $e^{*}$. Since $e^{*}$ in PVDF is proportional to the polarization $P_{O}\left(x_{s}\right)$ (to be discussed in Sec. (II B), the acoustic step response of the PPS method-after proper calibration-provides a simple means for directly determining the polarization profile in PVDF.

Therefore, by using the PPS method we can study the absolute magnitude of the polarization in the PVDF films both for the electron-beam-irradiated and for the nonirradiated regions, respectively, as well as for foils of different origin or different processing. This information on the comparative magnitude of the polarization is very useful for a clear understanding of the process of electron-beam poling in PVDF.

\section{H. EXPERIMERTAL METHODS}

\section{A. Electron-beam polng}

Unmetalized PVDF films, from different sources varying in thickness between 25 and $40 \mu \mathrm{m}$, were bonded onto a polished copper substrate and afterwards inserted into a conventional scanning electron nicroscope (Cambridge Mk 2A). All experiments were performed at room temperature at a pressure of $2 \times 10^{-5} \mathrm{mbar}$.

In general, a small area of the PVDF foil $\left(2 \mathrm{~mm}^{2}\right)$ was scanned by a focused electron beam at energies varying from 5 to $30 \mathrm{keV}$. A single scan of this area lasted $400 \mathrm{~s}$. The sweep rate of 100 lines/s resulted in an effective spot velocity of $0.16 \mathrm{~m} / \mathrm{s}$ across the sample.

Before the poling experiments the current of the incident electron beam was measured to be about $8 \times 10^{-10} \mathrm{~A}$ using a Faraday cup. During the poling process the curtent collected from the rear side of the PVDF film was also monitored.

The magnitude of the incident beam current as well as the irradiation time and the irradiated area, were optimized such as to create a large amount of polarization, but staying below the critical dose for the destruction of the specimen surface by chemical changes or by overheating. ${ }^{17}$ Immediately after poling we could demonstrate the piezoelectric activity of the irradiated PVDF films by generating ultrasound at $\mathrm{MHz}$ frequencies using the well-known pulse-echo technique. ${ }^{21}$

\section{Piezolectric pressur step (PPS) measurement}

We used a PPS system as recently described elsewhere. ${ }^{18,19}$ An acoustic rectangular pressure pulse of 1 -ns rise time and 100 -ns pulse duration is generated by the transient response of an $X$-cut-quartz disk of $3 \mathrm{~mm}$ thickness and $20 \mathrm{~mm}$ diameter to a rectangular pulse signal of $600 \mathrm{~V}$ and 100 ns. The repetition rate can be varied between 20 and 200 $\mathrm{Hz}$. The mechanical pulse is coupled to the PVDF film by a thin onl layer. A conducting rubber electrode of $3 \mathrm{~mm}$ diameter is pressed against the sample for the collection of the charge and the polarization-induced electrical current signals. These are amplified and directly displayed on a $1-\mathrm{GHz}$ oscilloscope. The pressure puise leading edge corresponds to a velocity or a pressure step wave, propagating in the sample. Space charges, polarization, or true piezoelectric distribu- tions (by changes of elementary dipole moments under strain) contribute to the observed corresponding time-dependent current signal. In the case of the piezoelectric polymer PVDF, having a finte conductance, the general quantitative expression ${ }^{18,19}$ reduces to the following relation between the signal current $I_{s}$ and the charge-compensated polarization:

$$
I_{s}(t)=\frac{A v}{l} e^{*}
$$

with

$$
e^{*}=P_{0}\left(x_{s}\right)-e_{33}\left(x_{s}\right)
$$

where $P_{0}\left(x_{s}\right)$ denotes polarization at the position $x_{s}$, $e_{33}\left(x_{s}\right)$ denotes other piezoelectric contributions, $A$ denotes cross section of the sample and sensing electrode, $v$ denotes acoustic particle velocity behind the pressure step, and $l$ denotes sample thickness (cf. Refs. 18 and 19).

It is important that the time constant defined by the capacity of the measuring electrode and the input impedance of the amplifier $(50 \Omega)$ is always kept below $1 \mathrm{~ns}$. For thin samples the electrode capacitance can be reduced by inserting-in series-an additional dielectric fim in front of the conducting rubber electrode. In the absolute evaluation of the piezoelectric properties we determined the constant $e^{*}$ with an experimental accuracy of $\pm 5 \%$. (For further details, including the calibration, see Refs. 18-20.) The dominant contribution to $e^{*}$ results from the local polarization $P_{0}\left(x_{s}\right)$ via the pure geometrical polarization change (i.e., the dipole density) under the step-wave deformation. All other contributions which are also proportional to $P_{6}\left(x_{5}\right)$ are contained in $e_{33}\left(x_{s}\right)$. These, in principle, can be ascribed to changes of the effective microscopic dipole moments in the polar crystallites including pyroelectric contributions due to the temperature increase within the pressure step, electrostriction (change in $\epsilon$ ), thermal libration, direct change of elementary dipole moments under stress, and others. The different mechanisms are well described and reviewed in the literature (de Reggi, ${ }^{22}$ Broadhurst et al., ${ }^{23}$ Kepler and Anderson, ${ }^{24}$ and Gerhard-Multhaupt, Gross, and Sessler ${ }^{25}$ ).

The lack of detailed microscopic data makes it difficult to predict the exact ratio or even the sign between $e_{33}\left(x_{s}\right)$ and $P_{0}\left(x_{s}\right)$ in the present case of uniaxial plane-wave deformation. We therefore compared $e^{*}$ with the polarization $P_{0}$ obtained from measurements of the charging and discharging currents in poling the sample first by high-voltage application and afterwards by discharging under zero-voltage condition. ${ }^{26}$ In these experiments the ratio between $P_{0}$ and $e^{*}$ amounted to 1.1 with an experimental error of $5 \% .{ }^{26} \mathrm{Ap}$ parently the different signal contributions mentioned above cancel each other under the deformation condition of the PPS method, thus leaving the dipole density term $P_{0}\left(x_{s}\right)$ as the dominant signal source, and therefore the signal current $I_{s}$ is a good measure for the polarization.

\section{RESULTS AND DISCUSSION}

The polarization of the PVDF films, which we observed after electron irradiation, was obtained for the following conditions. 
(a) The energy of the incident electrons was changed from 5 to $30 \mathrm{keV}$ in order to investigate the infuence of the electron penetration depth on the polarization.

(b) As will be described below, we found to our surprise that the polarization profile is strongly dependent also on the physical characteristics of the PVDF sample under investigation. Therefore we examined the polarization profiles of PVDF films of different origin and after different treatment but for identical, current, and voltage conditions in the scanning electron microscope (SEM).

(c) It was of special interest to us to measure the shape of the polarization profies for electron-beam poling and compare it with those obtained by other poling methods.

\section{A. Shape of the polarization proflles}

Due to the finite penetration of electrons into the sample we cannot expect a uniform distribution of the polarization across the film thickness. This fact is clearly demonstrated in Figs. 1(a)-1(d) which shows the polarization profles observed for the identical material (27- $\mu \mathrm{m}$ uniaxially stretched KF-polymer film) irradiated with focused electrons swept across an area of $2 \mathrm{~mm}^{2}$, beam current: $0.78 \mathrm{nA}$ at a rate of 100 Sines per second. The electron energy was $5,10,20$, and $30 \mathrm{keV}$, respectively. The PPS signals refer to the case that
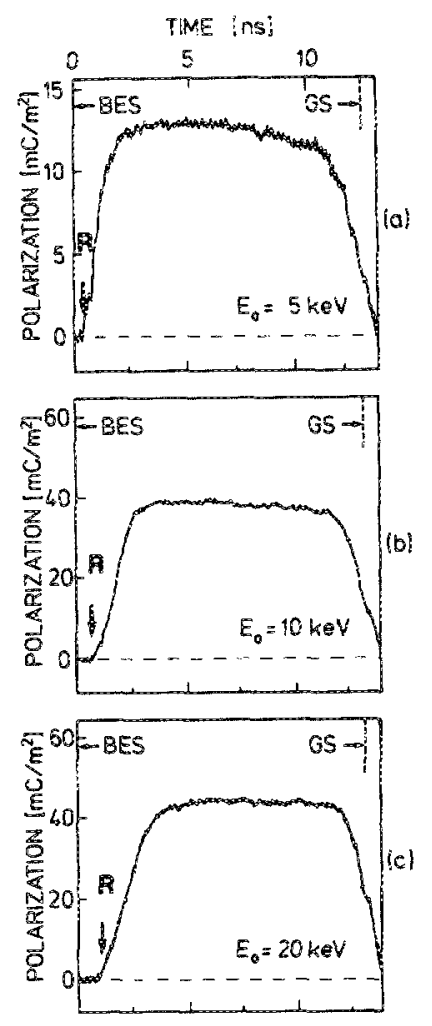

FIG. 1. Polarization profiles of uniaxially stretched $27-\mu \mathrm{m} \mathrm{KF}$. polymer films poled by electrons of different energies $E_{0}$. The polarization grows with increasing electron energy (see also Fig. 2). At the same time the profile become more asymmetric [see (c) and (d) ]. Note the different scale in (a).

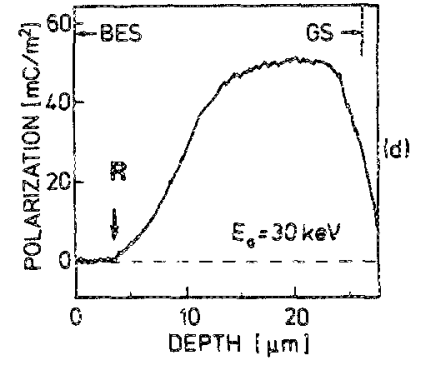

the acoustic step enters the PVDF sample from the same side from which the film surface had been irradiated by the poiarizing electron beam. In the following this surface will be called the beam-exposed ( $\mathrm{BE}$ ) surface. The polarization is seen to build up a few microns away from the BE surface inside the sample at a critical depth $R$ (see arrow). This starting depth $R$ grows when increasing the beam energy as is clearly evident from Figs. $1(a)-1(d)$. It must be emphasized, however, that generally $R$ is considerably smaller than the average penetration depth of electrons, which is rather more comparable to the range further inside the sample where the polarization exhibits its steepest rise.

In Fig. 1 (a) a different scale is used for the polarization. It is interesting to note (Fig. 2) that the maximum of the polarization inside the film grows with the beam voltage (for constant electron dose and for the same material), particulariy between 5 and $10 \mathrm{keV}$. Above $10 \mathrm{keV}$ the polarization still increases slightly with beam voltage, in our view because of the decreasing effective thickness of the polarized region, thereby increasing the effective field strength across the sample. This observation, however, shows the tendency of the polarization in $P V D F$ to saturate at high poling fields.

Furthermore, it is clearly visible in Fig. 1 that for increasing the energy of the polarizing electron beam the polarization profle becomes more asymmetric: The rising slope of the polarization becomes less steep and the plateau of maximurn polarization is displaced towards the rear side of the PVDF flm for the higher beam voltages. This effect is more pronounced for the samples irradiated by 20 - and $30-$ $\mathrm{keV}$ electrons, whereas for PVDF films polarized using electrons of only 5 and $10 \mathrm{keV}$ the profiles are rather symmetrical.

As already mentioned the energy of the polarizing electron beam is, however, according to our observation not the only parameter which determines the shape of the polarization distribution across the sample thickness. The polarization profiles differ strongly for materials of various sources, which have been shown to contain different fractions of $\alpha$ and $\beta$-phase crystallites, and consequently they also exhibit a different conductivity. ${ }^{27}$ This fact is clearly demonstrated in Fig. 3, showing the observed polarization profiles of two different samples poled under identical conditions: The up-

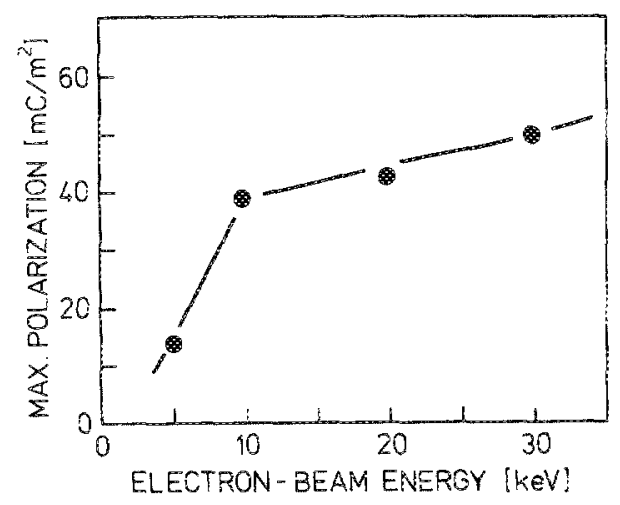

FIG. 2. The maximum polarization inside the sample for constant beam current as a function of electron-beam energy. For other details see fig. 1 . 

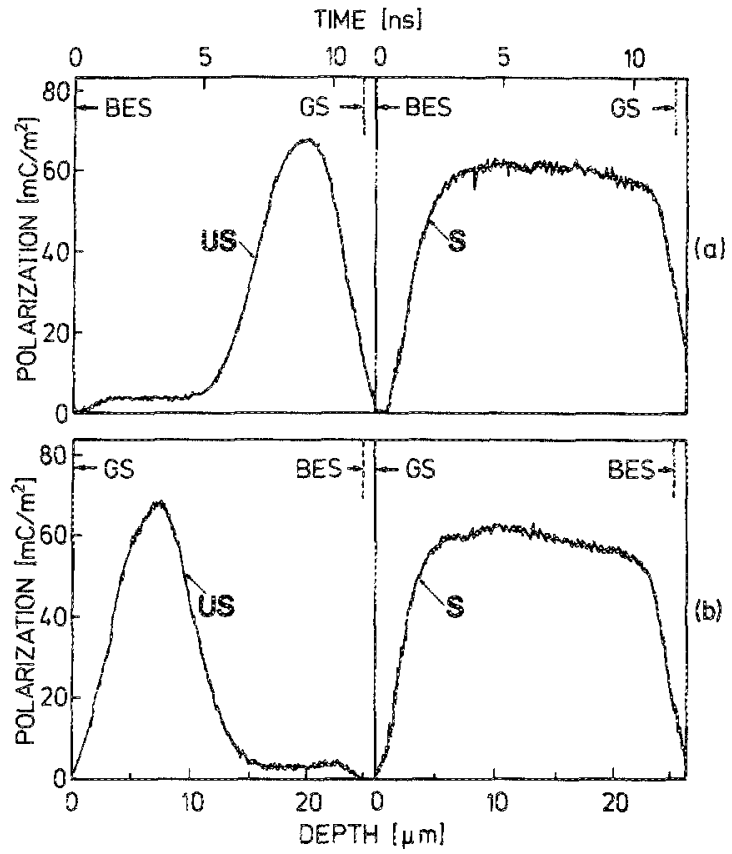

FIG. 3. PPS response of two diferent PVDF fims irradiated with 10-keV electrons under identical poling conditions. Left-hand side: profles for an unstretched $25 \mu \mu \mathrm{m}$ KF polymer film (US). Right-hand side: profiles obtained for biaxially stretched $25-\mu \mathrm{m}$ Solef flm (S). The upper (a) and the lower curves (b) difer only in that the pressure step enters from opposite surfaces of the sample.

per two curves in Fig. 3(a) show profiles which were both measured by PPS in such a way that the acoustic transducer for the generation of the pressure pulse was adjacent to the BE surface. Vice versa, for the measurement of the lower two curves in Fig. $3(0)$ the piezoelectric transducer was in contact with the grounded rear electrode which is called the $G$ surface (GS) in the following. In this way we could demonstrate the reliability of our observation of asymmetric shapes.

Let us now look at Figs. 3(a) and $3(b)$ in more detail. They represent observed profiles from two different samples, called US (unstretched) and S (stretched), respectively, which were polled by electron irradiation under identical conditions. The two left curves refer to an unstretched PVDF foil (US) from Kureha $25 \mu \mathrm{m}$ thick, containing a mixture of $\alpha$-and $\beta$-phase crystallites and showing a relatively high electrical conductivity under the infuence of electron beam while the two curves on the right are taken from a biaxially stretched (S) $25-\mu \mathrm{m}$ Solvay flim, which consists of nearly pure $\beta$-PVDF and shows lower electrical conductivity. ${ }^{27}$

Clearly, the unstretched sample (US) on the left in Fig. 3 shows a narrow profile which is displaced of center towards the grounded $G$ surface (GS). It is interesting that the two samples (US) and (S) represented in the left-and right-hand sides of Fig. 3, respectively, exhibit such different polarization profiles in spite of having been polarized under identical conditions. In particular, it is exactly those PVDF films that already show a highly asymmetric piezoelectric activity for thermal and room-temperature poling ${ }^{21,28}$ which are also the most asymmetric under electron-beam polariza-

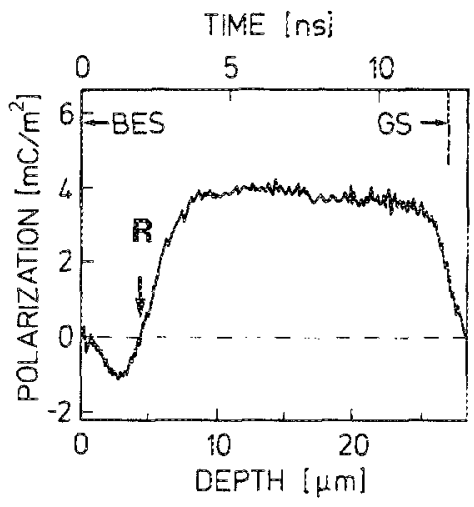

tion. This fact clearly demonstrates that for the asymmetry of the profiles the material parameters of the foil are at least as important as the process of electron-beam polarization. The electrical conductivity of the samples which grows with increasing $\alpha$-phase content seems to be an important parameter for the shape of the profiles and the homogeneity of the polarization. Mobile charge carriers, the nature of which is still anknown, seem to have a considerable infiuence on the poling process and on the final profile of the polarization.

There is another interesting feature which we have observed: In Fig. 4 let us focus our attention on the region between the depth $R$ (onset of the polarization) and the BE surface. A small amount of polarization pointing into the direction opposite to the main polarization discussed so far has been observed. This surprising effect of "opposite polarization" (shown in Fig. 4) appeared in most of the samples investigated so far and could best be seen under the conditions of weak polarization. We believe that this "reverse polarization" can be attributed to the reverse electric field existing between the negative space charge of the injected electrons, at a deptín of a few microns, and a positive surface layer, about $10 \mathrm{~nm}$ deep, adjacent to the BE surface. In our view these positive charges close to the $\mathrm{BE}$ surface are generated by the secondary-electron emission from the contactfree beam-exposed surface.

Referring to the sample- and beam-dependent asymmetries of the polarization profiles, shown in Figs. 1 and 3 , as well as to the effect of reverse polarization near the BE surface shown in Fig. 4 , we propose the following qualitative picture of the poling process occurring during electron irradiation.

As the electrons penetrate into the film (up to a few microns dependent on their energy), they create a virtual negative electrode located at the average penetration depth. Between this virtual electrode and the grounded rear electrode of the film the main poling field bulds up, apparently modifed by the electrical conductivities in the polymer samples. Under the influence of this effective electric field and dependent on the dielectric properties of the film dipolar orientation takes place in the ferroelectric sample, i.e., the main polarization builds up.

Between the main (negative) space-charge region and the weaker positive charges near the BE surface a secondary electric field of reverse direction is rormed as pointed out above. It remains rather small by comparison with the main 
field perhaps because of the increased electrical conductivity in the irradiated region-often referred to as "radiation-induced conductivity" (RIC). ${ }^{9}$ But the resulting field seems to be high enough to orient the molecular dipoles to a certain degree. The RIC is not strong enough to completely suppress the occurrence of the reverse polarization shown in Fig. 4 .

Under the infuence of electron bombardment the charge carriers in EVDF are known to acquire a relatively high mobility," particularly when the beam energy is large. Driven by the electric fields of the virtual negative electrode the charge carriers are drifting easily towards the grounded rear electrode and also towards the BE surface. The growing asymmetry of the profiles in Figs. $1(c)$ and $1(d)$ as well as the larger polarization for the higher beam energies (see Fig. 2) can perhaps be accounted for by such a radiation-induced (or thermally activated) motion of the virtual electrode towards the $G$ surface.

In contrast, PVDF samples having been poled by electrons of only 5 or $10 \mathrm{keV}$, which penetrate into the sample less than one-tenth of the whole sample thickness, are observed to show nearly symmetrical profiles. These samples are known to have a high $\beta$-phase content (sample marked $s$ in Fig. 3 on the left-hand side). The electrical conductivity for oriented PVDF flims is known to be more than one order of magnitude smaller than in the unoriented state. ${ }^{29}$ We believe that in stretched PVDF samples the charge carriers cannot move easily towards the $G$ surface during the irradiation with low-energy electrons, and therefore a more uniform poling field and polarization builds up. For the unstretched sample on the other hand, marked US in Fig. 3, which probably contains a large proportion of $\alpha$-phase crystallites, the poling process is more complicated. Here an $\alpha \rightarrow \delta$ phase transition occurs as we observed in $\mathrm{x}$-ray-diffaction experiments, ${ }^{27}$ which agree well with the results of $\mathrm{Da}$ vis et $a l^{30}$ and Newman et $a l^{31}$ for corona-poled PVDF containing a mixture of $\alpha$ - and $\beta-\mathrm{PVDF}$. We assume a poling mechanism which is basically similar to the process of thermal poling and which has already been described elsewhere. ${ }^{28,32-34}$

It has been concluded by Lang et $a ._{.3}{ }^{35}$ as well as by Mopsik and DeReggi ${ }^{36}$ from thermal pulse experiments that

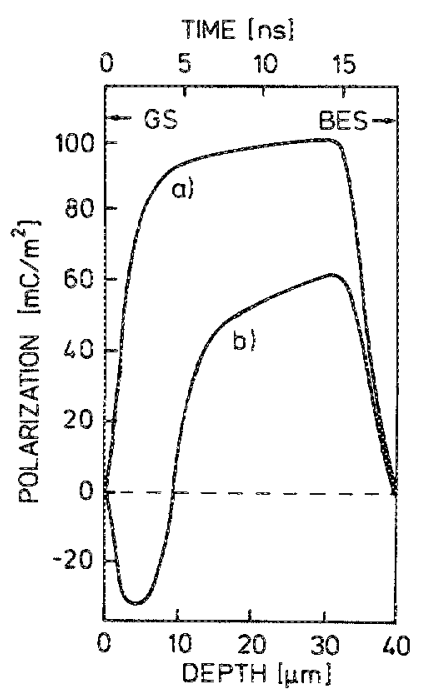

FIG. 5. Production of a bimorph by electron irradiation of $\mathrm{PVDF}$ films. (a) Profile of the commercially polarized $40-\mu \mathrm{m}$ Yarsley film; (b) profile of the same film after irradiation with $30-\mathrm{keV}$ electrons in the reverse direction resulting in a partially reversed polarization and in a reduction of the original polarization. polarization properties across the film may be related to inherent inhomogeneities in the samples. Finally, we cannot completely exclude that fact being responsible-at least partially-for our observed profles.

It should also be mentioned that electron-beam-polarized PVDF films produce PPS signals, which are quite different from those obtained for electret materials such as polyfuoroethylenepropylene (FEP) and polyethyleneterephtalate (PETP) charged by a 20 - or $30-\mathrm{keV}$ electron beam, but with a charge density which is more than 2 orders of magnitude below that used in our experiments. ${ }^{37}$ The much higher charge storage capability of $F V D F$ is regarded as a consequence of the strong charge-compensated polarization in this matera!. In FEP and PETP the electrons entering the foil during the irradiation are trapped at the penetration depth and no further motion seems to occur even if the sample is, afterwards, heated up to $150^{\circ} \mathrm{C}$.

\section{Bimorph generation by electron-beam poling}

The finite penetration of electrons into the PVDF fim during the electron irradiation is very useful for the production of piezoelectric bimorphs. Bimorphs are used for a wide variety of applications mostly based on piezoelectric fexure mode devices. ${ }^{38}$

In order to generate a bimorph we used a commercially polarized 40- $\mu \mathrm{m}$-thick PVDF film (from Yarsley Technical Centre, $\mathrm{L}$ td), the positive sutface of which was exposed to an electron beam of $30 \mathrm{keV}$. In Fig. 5 the polarization distribution before (curve a) and after (curve b) the electron irradiation is shown. The polarization profles have been measured only for the case that the pressure step enters the sample from the $G$ surface. From curve b of Fig. 5 it can be seen that near the $G$ surface the polarization is reversed, and near the BE surface the polarization is reduced. The fact that the polarization remains partly in its initial direction up to a depth exceeding the penetration depth for $30-\mathrm{keV}$ electrons seems to us to support the poling model for $\beta$-phase PVDF described above.

\section{Poing of VDF-TrFE copolymar by electron hrodation}

The poling method described in this paper cannot only be applied to pure PVDF, but also allows the poling of its copolymers with $\operatorname{TrFE}$. This was demonstrated by irradiating a solution grown $60 / 40$ vinylideneffuride-trifluoroethylene (VDF-TrFE) copolymer sample by $20-\mathrm{keV}$ electrons. The resulting polarization profile is shown in Fig. 6 . It can be seen that the amount of polarization of the copolymer is weaker than for PVDF (cf. Fig. 1) and that its profile is asymmetrically displaced towards the $G$ surface. The same behavior was observed for a VDF-TrFE copolymer film of composition $75 / 25$.

The reason for the relatively weaker polarization of the copolymer is not yet understood. Lovinger ${ }^{39}$ has reportedinduced by $100-\mathrm{keV}$ electrons at a charge density of more than $4 \mathrm{C} / \mathrm{m}^{2}-$ ferroelectric to paraelectric phase transition to occur in the VDF-TrFE copolymers. In our own preliminary $x$-ray-diffraction experiments we did, however, observe no difference in the diffraction pattern of unirradiated co- 


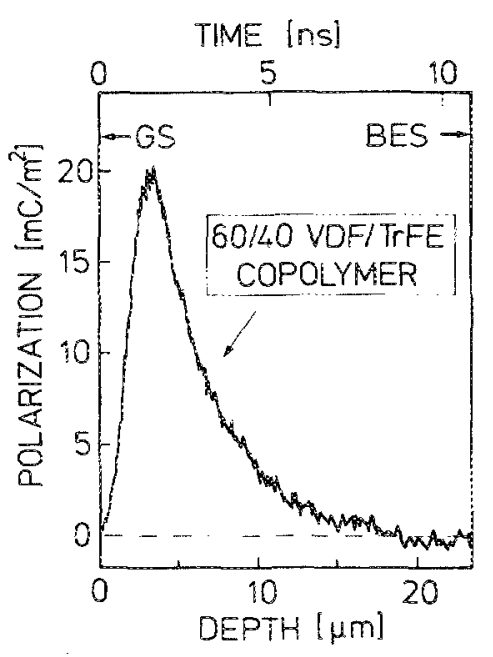

polymer films compared with samples irradiated by 20 - and $30-\mathrm{keV}$ electrons, respectively. But the electron doses used in our experiments were more than 1 order of magnitude below those quoted by Lovinger.

Our $\mathrm{x}$-ray diffraction results, however, indicate that copolymers grown from a solution (acetone) contain a certain fraction of a paraelectric crystalline phase. Thus the rather asymmetric polarization profile in electron-beam-polarized 60/40 VDF-TrFE copolymer may be a conseguence of partly disordered crystal phases in solution-grown copolymer films. Moreover, we could show that the electric conductivity of the copolymer samples are of the same magnitude as for the sample whose profile is shown on the left-hand side of Fig. 3 having considerable $\alpha$-phase content. For a fuller understanding of this behavior more experiments are necessary.

\section{CONCLUSIONS}

It has been shown that the irradiation of PVDF films by a focused electron beam is a useful tool for creating a wide variety of polarization profiles with a high lateral resolution in PVDF fims. Using the PPS method for examining the polarization profiles of the electron-beam-poled RVDF fims, we have demonstrated that the polarization is strongly dependent on the electron energy. The polarization buils up at a characteristic deptl $R$ below the beam exposed surface, rising when the electron energy is increased and at the same time becoming more asymmetric as a consequence of the increasing electronic penetration depth. Due to the finite penetration of the electrons into the sample a strong polarizing electric field builds up between the negative charge cloud and the positive image charges in the grounded metal electrode at the rear of the sample.

The polarization profile is found to depend not only on the beam energy but equally strongly on the materiai parameters. ${ }^{28,35,36}$

For samples of high $\alpha$-phase content and of a relatively high electrical conductivity, we observed a very asymmetric distribution of the polarization, the maximum of which is displaced towards the grounded rear electrode. These asymmetric profiles-often referred to as "thermal profles"-are observed for exactly those specimens which are known to show a highly nonuniform piezoelectric activity with a maximum always close to the positive poling electrode. ${ }^{21,28}$

For samples of pure $\beta$-PVDF we observed a small amount of reverse polarization (opposite to the main polarization) between the beam-exposed surface and the depth $R$ where the main polarization starts inside the film. We believe that secondary emission from the beam-exposed surface is responsible for the small reverse-poling field near the front surface.

Electron-beam poling of VDF-TrFE copolymer films leads to a much smaller and asymmetric polarization. The reason for this behavior is not yet understood.

As far as applications are concerned, electron-beam poling of PVDF foils can be used for the production of welldefined piezoelectric monomorphs ${ }^{16}$ and bimorphs for flexure mode devices as pointed out previously.

When using a focused electron beam for the poling process, in addition, very small domains can be poled in PVDF. A focused electron beam may therefore be useful for fast data storage in ferroelectric materials. The spatial resolution is only limited by the width of the electron diffusion inside the sample. For the read-out process a weaker electron beam can be used with or without pyroelectric enhancement of the surface charges.

\section{ACKNOWLEDGMENTS}

Two of us (D. S. and K. D.) gratefully acknowledge the stimulating discussions with Professor E. Fukada, Professor G. Kämpf, and Dr. R. Pott. We also express our gratitude to the Bayer $\mathrm{AG}$, Leverkusen for the gift of a scanning electron microscope and partial financial support of the project. Three of us (E. B., K. H., and W. E.) are grateful to the Deutsche Forschungsgemeinschaft for financial support.

'H. Sussner and K. Dransfeld, Colloid Polym. Sci. 257, 591 (1979).

${ }^{2}$ G. M. Sessler, J. Acoust. Soc. Am. 70, 1596 (1981).

${ }^{3}$ M. G. Broadhurst and G. T. Davis, in Electrets, Topics in Applied Physics, Vol. 33, edited by G. M. Sessler (Springer, Berlin, 1980), p. 285.

${ }^{4} A$. J. Lovinger, in Developments in Crystaline Polymers-l, edited by $\mathrm{D}$. C. Basset (Applied Science, London, 1982), p. 195.

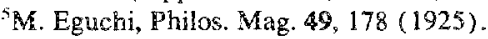

'H. Kawai, Jpn. J. Appl. I2hys. 8, 975 (1969).

${ }^{7}$ P. D. Southgate, Appl. Phys. Lett. 28, 250 (1976).

${ }^{8}$ G. M. Sessler, R. Gerhard-Multhaept, J. E. West, and A. Berraissoul, in Proceedings of the 2nd International Symposium on Nondestructive Characterization of Materials, Montreal, 1986, edited by J. F. Bussiere (Plenum, New York, 1987).

"B. Gross, 11. y. Seggern, and R. Gerhard-Multhanpt, J. Phys. D 18, 2497 (1985).

${ }^{10}$ B. Gross, R. Gerhard-Multhaupt, A. Berraissoul, and G. M. Sessler, J. Appl. Phys. 62, 1429 (1987).

G. M. Sessler, J. E. West, and R. Gerhard, Polym. Bull. 6, 109 (1981).

'G. M. Sessier, J. E. West, and R. Gerhard, Phys. Rev. Lett. 48,563 (1982).

${ }^{13}$ R. Gerhard-Multhaupt, Phys, Rev. B 27, 2494 (1983).

${ }^{14 D}$ W. W. Byatt, U. S. Patent No. 4,059,827 (1977).

$15 \mathrm{O}$. Schilling and K. Dransfeld, Ferroelectrics 81,377 (1988). 
IfE. Fukada, G. M. Sessler, J. E. West, A. Beraissoul, and P. Günther, J. Appl. Phys. 62, 3643 (1987).

17L. Reimer, in Fhysical Aspects of Electron Microscopy and Microbeam Anaiysis, edited by B. M. Siegel and D. R. Beaman (Wiley, New York, 1975), p. 231.

${ }^{1}$ W. Eisenmenger and M. Haardt, Solid State Commun. 41,917 (1982).

${ }^{19}$ M. Haardt and $W$. Eisenmenger, IEEE 1982 Annual Report (CEIDP)(IEEE, Piscataway, NJ, 1982), p. 46.

${ }^{31}$ R. Gerhard-Multhaupt, G. M. Sessler, J. E. West, K. Holdik, M. Haardt, and W. Eisenmenger, J. Appl. Phys. 55, 2769 (1984).

${ }^{21} \mathrm{H}$. Sussner and K. Dransfeld, J. Polym. Soi. Polym. Phys. Ed. 16, 529 (1978).

${ }^{22}$ A. S. DeReggi, Ferroelectries 60, 83 (1984).

${ }^{23}$ M. G. Broadhurst, G. T. Davis, J. E. Mckinney, and R. E. Collins, 3 Appl. Phys. 49, 4992 (1978).

${ }^{24}$ R. G. Keppler and R. A. Anderson, CRC Crit. Rev. Solid State Mater. Sci. 9, 399 (1980).

${ }^{25}$ R. Gerhard-Muthaup, B. Gross, and G. M. Sesster, in Electrets, 2nd ed., Topics in Applied Physics, Vol. 33, edired by G. M. Sessler (Springer, Berlin, 1987).

${ }^{26} \mathrm{M}$. Womes, Dipioma thesis, University of Stutgart, 1988 (unpublished).
${ }^{27}$ D. Schilling, Ph. D. thesis, University of Konstanz, 1988 (HartungGorre, Konstant, 1988).

${ }^{24} \mathrm{~K}$. Holdik, Ph. D. thesis, University of Stuttgart, 1985.

${ }^{29}$ A. J. Bur, Polymer 22, 1288 (1981)

${ }^{36}$ G. T. Davis, J. E. McKinney, M. G. Broadhurst, and S. C. Roth, J. Appl. Phys. 49, 4998 (1978).

${ }^{31}$ B. A. Newman, C. H. Yoon, K. D. Pae, and J. I. Scheinbeim, J. Appl. Phys. 50, 6095 (1979).

${ }^{3}$ W. Eisenmerger, M. Haardt, and $\mathrm{K}$. Holdik, IEKE 1982 Annual Report (CERDP) (IEEE, Piscataway, NJ, 1982), p. 52.

${ }^{3}$ M. Haardt, Dh. D. thesis, University of Stuttgart, 1982.

${ }^{34} \mathrm{~K}$. Holdik and W. Eisenmenger, in Proceedings of the 5 th International Symposiam on Electrets, Heidelberg, 7985 , edited by G. M. Sessler and R. Gerhard-Multhapt (IENE, Piscataway, ND, 1985), p. 553.

${ }^{35}$ S. B. Lang, A. S. DeReggi, F. J. Mopsik, and M. G. Broadhurst, J. Appl. Phys. 54, 5598 (1983).

${ }^{76}$ F. J. Mopsik and A. S. DeReggi, Appl. Phys. Lett. 44, 65 (1984)

${ }^{17} \mathrm{R}$. Gerhard-Multhaupt, M. Haardt, W. Eisenmenger, and G. M. Sessier, J. Phys. D 16, 2247 (1983).

${ }^{38}$ M. A. Marcus, Ferroelectrics 57, 203 (1984).

${ }^{36}$ A. J. Lovinger, Macromolecules 18,910 (1985). 